\title{
On a degenerate boundary value problem
} to the two-dimensional self-similar nonlinear wave system

\author{
Jiajia Liu', Yanbo Hu* and Tiehong Zhao ${ }^{1}$
}

\section{"Correspondence:}

yanbo.hu@hotmail.com 'Department of Mathematics, Hangzhou Normal University, Hangzhou, P.R. China

\begin{abstract}
This paper focuses on a degenerate boundary value problem arising from the study of the two-dimensional Riemann problem to the nonlinear wave system. In order to deal with the parabolic degeneracy, we introduce a partial hodograph transformation to transform the nonlinear wave system into a new system, which displays a clear regularity-singularity structure. The local existence of classical solutions for the new system is established in a weighted metric space. Returning the solution to the original variables, we obtain the existence of classical solutions to the degenerate boundary value problem for the nonlinear wave system.
\end{abstract}

MSC: 35L65; 35L80; 76H05

Keywords: Nonlinear wave system; Degenerate hyperbolic; Characteristic decomposition; Classical solution

\section{Introduction}

We are interested in a degenerate boundary value problem arising from the study of the two-dimensional four-constant Riemann problem to the nonlinear wave system

$$
\left\{\begin{array}{l}
\rho_{t}+(\rho u)_{x}+(\rho v)_{y}=0, \\
(\rho u)_{t}+p_{x}=0, \\
(\rho v)_{t}+p_{y}=0
\end{array}\right.
$$

where $\rho,(u, v)$, are, respectively, the density and the velocity, and $p=p(\rho)$ is a given function of $\rho$. This system is obtained either by ignoring the quadratic terms in the velocity $(u, v)$ to the two-dimensional isentropic compressible Euler equations in the gas dynamics, or by writing the nonlinear wave equation as a first-order system. We refer the reader to references $[3,4]$ for the background information.

The multidimensional Riemann problem of the quasilinear hyperbolic conservation laws is one of important problems in mathematical fluid dynamics containing in particular the oblique shock reflection problem and the dam collapse problem. Most importantly, the Riemann problem performs the role of 'building blocks' for all fields of theory, numerics, and applications, see the survey [18] and the references therein. The study of 
the two-dimensional Riemann problem to the Euler equations was initiated by Zhang and Zheng [32]. The authors provided a set of conjectures on the structure of solutions by using the generalized characteristic analysis method and numerical experiments. However, until now, none of them has been completely proved due to the existence of transonic structures $[7,19,36]$. Many pieces of work have been contributed on understanding these transonic structures for the Euler equations and its related systems. We refer the reader to $[17,20-23,25-27,30]$ and references cited there, and especially the monographs $[19,36]$ for the results of the Euler and pressure-gradient systems. In particular, for the relevant results of the two-dimensional Riemann problem to the nonlinear wave system (1.1), one may consult $[6,10-16]$ and references therein.

We consider in this paper system (1.1) together with a smooth state function $p=p(\rho)$ satisfying

$$
\forall \rho>0, \quad p(\rho) \gtrless 0, \quad p^{\prime}(\rho)>0, \quad \text { and } \quad p^{\prime \prime}(\rho) \gtrless 0 .
$$

It is clear that the well-known equations of state of the polytropic gas $p=A \rho^{\gamma}(\gamma>1)$ and of the Chaplygin gas $p=-1 / \rho$ satisfy (1.2). The equation of state of the Chaplygin gas was introduced by Chaplygin [5] and was taken as a suitable mathematical approximation for calculating the lifting force on a wing of an airplane in aerodynamics by Tsien [28] and von Karman [29]. Moreover, this equation of state has been advertised as a possible model for dark energy; see, e.g., [2, 8]. We are looking for the self-similar solutions of (1.1), that is, the solutions depend only on the self-similar variables $(\xi, \eta)=(x / t, y / t)$. In terms of variables $(\xi, \eta)$, system $(1.1)$ can be transformed to

$$
\left\{\begin{array}{l}
-\xi \rho_{\xi}-\eta \rho_{\eta}+(\rho u)_{\xi}+(\rho v)_{\eta}=0 \\
-\xi(\rho u)_{\xi}-\eta(\rho u)_{\eta}+p_{\xi}=0, \\
-\xi(\rho v)_{\xi}-\eta(\rho v)_{\eta}+p_{\eta}=0 .
\end{array}\right.
$$

The eigenvalues of (1.3) are

$$
\Lambda_{0}=\frac{\eta}{\xi}, \quad \Lambda_{ \pm}=\frac{\xi \eta \pm \sqrt{p^{\prime}(\rho)\left(\xi^{2}+\eta^{2}-p^{\prime}(\rho)\right)}}{\xi^{2}-p^{\prime}(\rho)} .
$$

We see from (1.2) and (1.4) that system (1.3) is hyperbolic at the infinity (i.e., $|\xi|+|\eta|=\infty$ ) and changes type to elliptic near the origin. The hyperbolic and elliptic regions may be separated by a boundary curve composed by degenerate curves and shocks, which are free boundaries to be determined together with the solutions [1]. To construct a global solution of nonlinear mixed-type system, iterative methods seem to be the most likely choices.

The purpose of the present paper is to establish the local existence of classical solutions to the two-dimensional self-similar nonlinear wave system (1.3) with degenerate boundary data, which is an essential step for using an iterative process to construct a global solution of mixed type equation. The local existence of classical sonic-supersonic solutions was investigated for compressible Euler equations with polytropic gases in $[9,33,35]$ and for a pressure-gradient system in [34]. We consider the degenerate boundary value problem of (1.1) with the convex equation of state satisfying (1.2) in the current paper and will explore that problem for the general nonconvex equation of state in the next work. 
The rest of the paper is organized as follows. In Sect. 2, we describe the problem in detail and then state our main result. Section 3 is devoted to reformulating the problem in the new dependent and independent variables. In Sect. 4, we complete the proof of the main results by solving the new problem and then converting the solution to the original coordinates.

\section{The problem and the main results}

We first decouple $p$ from $(\rho u)$ and $(\rho v)$ to obtain a second-order quasilinear equation,

$$
\left(a(p)-\xi^{2}\right) p_{\xi \xi}-2 \xi \eta p_{\xi \eta}+\left(a(p)-\eta^{2}\right) p_{\eta \eta}+b(p)\left(\xi p_{\xi}+\eta p_{\eta}\right)^{2}-2\left(\xi p_{\xi}+\eta p_{\eta}\right)=0
$$

where

$$
a(p)=\frac{1}{\rho^{\prime}(p)}>0, \quad b(p)=-\frac{\rho^{\prime \prime}(p)}{\rho^{\prime}(p)}=\frac{a^{\prime}(p)}{a(p)}
$$

for all $p>0$ by (1.2). The two eigenvalues of (2.1) are

$$
\tilde{\Lambda}_{ \pm}=\frac{\xi \eta \pm \sqrt{a(p)\left(\xi^{2}+\eta^{2}-a(p)\right)}}{\xi^{2}-a(p)} .
$$

For convenience to deal with our problem, we rewrite (2.1) in terms of the polar coordinates $(r, \theta)$ as

$$
P_{\theta \theta}-\frac{r^{2}\left(r^{2}-a(P)\right)}{a(P)} P_{r r}+r P_{r}+\frac{b(P) r^{3}}{a(P)} P_{r}^{2}-\frac{2 r^{2}}{a(P)} P_{r}=0
$$

where $r=\sqrt{\xi^{2}+\eta^{2}}, \theta=\arctan (\eta / \xi)$ and $P(r, \theta)=p(r \cos \theta, r \sin \theta)$. The two family of characteristics are defined as

$$
\Gamma_{ \pm}: \frac{d r}{d \theta}= \pm \lambda, \quad \lambda=\sqrt{\frac{r^{2}\left(r^{2}-a(P)\right)}{a(P)}} .
$$

It is clear that Eq. (2.4) is of mixed type: hyperbolic for $r^{2}>a(P)$, elliptic for $r^{2}<a(P)$ and parabolic degenerate for $r^{2}=a(P)$.

Let $r_{a}<r_{b}$ be two positive constants and $\Gamma: \theta=\varphi(r)$ be a smooth curve defined on $\left[r_{a}, r_{b}\right]$ satisfying $\left|\varphi^{\prime}(r)\right| \leq \varphi_{0}$ for same positive constant $\varphi_{0}$. That means the curve $\Gamma$ can not be a circular arc. We assign the boundary data on $\Gamma$ as follows:

$$
P(\varphi(r), r)=P_{0}(r), \quad P_{\theta}(\varphi(r), r)=P_{1}(r) \quad \text { with } a\left(P_{0}(r)\right)=r^{2}
$$

The aim of the paper is to look for a classical solution of the boundary value problem (2.4) (2.6). Since the wave speed $\lambda=0$ on $\Gamma$, the hyperbolic problem (2.4) (2.6) is parabolic degenerate.

In the hyperbolic region, Eq. (2.4) has the interesting characteristic decomposition [10]

$$
\left\{\begin{array}{l}
\partial_{+} \partial_{-} P=Q\left(\partial_{+} P-\partial_{-} P\right) \partial_{-} P, \\
\partial_{-} \partial_{+} P=Q\left(\partial_{-} P-\partial_{+} P\right) \partial_{+} P,
\end{array}\right.
$$


where

$$
\partial_{ \pm}:=\partial_{\theta} \pm \lambda \partial_{r}, \quad \text { and } \quad Q=\frac{a^{\prime}(P) r^{2}}{4 a(P)\left(r^{2}-a(P)\right)} .
$$

Introduce

$$
R=\partial_{+} P, \quad S=\partial_{-} P,
$$

from this and (2.5) we have

$$
P_{\theta}=\frac{R+S}{2}, \quad P_{r}=\frac{R-S}{2 \lambda}=\frac{\sqrt{a(P)}}{2 r} \cdot \frac{R-S}{\sqrt{r^{2}-a(P)}} .
$$

Moreover, from (2.7), we obtain the system for $(P, R, S)$

$$
\left\{\begin{array}{l}
P_{\theta}=\frac{R+S}{2}, \\
R_{\theta}-\lambda R_{r}=\frac{a^{\prime}(P) r^{2} R}{4 a(P)} \cdot \frac{S-R}{r^{2}-a(P)}, \\
S_{\theta}+\lambda S_{r}=\frac{a^{\prime}(P) r^{2} S}{4 a(P)} \cdot \frac{R-S}{r^{2}-a(P)},
\end{array}\right.
$$

where $\lambda$ is defined in (2.5). Then we look for a local classical solution of system (2.9) with the boundary data

$$
(P, R, S)(\varphi(r), r)=\left(P_{0}(r), P_{1}(r), P_{1}(r)\right), \quad \forall r \in\left[r_{a}, r_{b}\right]
$$

We point out that the local existence of the degenerate boundary value problem (2.9) (2.10) cannot be obtained directly by the classical local existence theory of nonlinear hyperbolic equations (see, e.g. [24, 31]). This is because system (2.9) is not a continuously differentiable system by the degeneracy. The idea we employed here is inspired by the work of Zhang and Zheng [33] for studying the steady Euler equations. The main technique is to isolate possible singularities by introducing a partial hodograph transformation. We establish the local existence and uniqueness of classical solutions for a new system under a suitable function class by using the fixed point method. Converting the solution to the original coordinates, we thus obtain a local classical solution to the problem (2.9) and (2.10). The results of this paper can be stated as follows.

Theorem 1 Suppose that the equation of state $p=p(\cdot) \in C^{4}$ satisfies (1.2). Moreover, we assume the functions $\left(\varphi, P_{0}, P_{1}\right)(r)$ satisfy

$$
\begin{aligned}
& \varphi(r) \in C^{4}\left(\left[r_{a}, r_{b}\right]\right) \quad \text { with }\left|\varphi^{\prime}(r)\right| \leq \varphi_{0}, \\
& P_{0}(r) \in C^{4}\left(\left[r_{a}, r_{b}\right]\right), \quad P_{1}(r) \in C^{3}\left(\left[r_{a}, r_{b}\right]\right) \quad \text { with }\left|P_{1}(r)\right| \geq k_{0},
\end{aligned}
$$

where $\varphi_{0}$ and $k_{0}$ are two positive constants. Then the degenerate boundary value problem (2.9) (2.10) has a classical solution in the hyperbolic region near $\Gamma$.

From Theorem 1, we have the following.

Theorem 2 Let the assumptions in Theorem 1 hold. Then there is a classic solution to the degenerate boundary value problem (2.4) and (2.6) in the hyperbolic region near $\Gamma$. 


\section{The problem in new coordinates}

In this section, we introduce new dependent and independent variables to reformulate the problem. To deal with the singularities caused by the degenerate, we first introduce a partial hodograph transformation as follows:

$$
t=\sqrt{r^{2}-a(P(r, \theta))}, \quad \tilde{r}=r
$$

Note that the sonic curve $\Gamma$ is transformed to a segment on $t=0$ with $\tilde{r} \in\left[r_{a}, r_{b}\right]$. From (3.1) one has

$$
\partial_{\theta}=-\frac{a^{\prime}(P)(R+S)}{4 t} \partial_{t}, \quad \partial_{r}=\partial_{\tilde{r}}+\frac{4 r^{2} t-a^{\prime}(P) \sqrt{a(P)}(R-S)}{4 r t^{2}} \partial_{t} .
$$

In terms of $(t, \tilde{r})$, system (2.9) can be rewritten as

$$
\left\{\begin{array}{l}
R_{t}+\frac{2 r t^{2}}{a^{\prime}(P) \sqrt{a(P)} S+2 r^{2} t} R_{r}=\frac{a^{\prime}(P) r^{2} R}{2 \sqrt{a(P)}\left[a^{\prime}(P) \sqrt{a(P)} S+2 r^{2} t\right]} \cdot \frac{R-S}{t}, \\
S_{t}-\frac{2 r t^{2}}{a^{\prime}(P) \sqrt{a(P)} R-2 r^{2} t} S_{r}=\frac{a^{\prime}(P) r^{2} S}{2 \sqrt{a(P)}\left[a^{\prime}(P) \sqrt{a(P)} R-2 r^{2} t\right]} \cdot \frac{S-R}{t},
\end{array}\right.
$$

together with a decoupled trivial equation

$$
\partial_{t} P=-\frac{2 t}{a^{\prime}(P)} .
$$

Here and below we still use $r$ to represent $\tilde{r}$ and denote $P(t, r)=P(r, \theta), R(t, r)=R(r, \theta)$, $S(t, r)=S(r, \theta)$, which will not cause confusion in understanding. Furthermore, we find by (1.2) that

$$
a\left(P_{0}(r)\right) \geq k_{1}>0, \quad\left|a^{\prime}\left(P_{0}(r)\right)\right| \geq k_{2}>0, \quad \forall r \in\left[r_{a}, r_{b}\right],
$$

for some positive constants $k_{1}, k_{2}$. Hence we can solve $P(t, r)$ from Eq. (3.4) with the initial data $P(0, r)=P_{0}(r)$. That means system (3.3) is closed in the coordinates $(t, r)$.

Corresponding to the boundary data (2.10), the initial data of (3.3) are

$$
(R, S)(0, r)=\left(P_{1}(r), P_{1}(r)\right), \quad \forall r \in\left[r_{a}, r_{b}\right] .
$$

In addition, by (2.6) we see that

$$
P_{r}(\varphi(r), r)=P_{0}^{\prime}(r)-\varphi^{\prime}(r) P_{1}(r):=P_{2}(r),
$$

which along with (3.3) yields

$$
\left(R_{t}, S_{t}\right)(0, r)=\left(P_{2}(r),-P_{2}(r)\right), \quad \forall r \in\left[r_{a}, r_{b}\right],
$$

for smooth solutions. Therefore, we look for a classic solution to system (3.3) with the initial data (3.6) and (3.7).

Next we introduce two new dependent variables to homogenize the initial data,

$$
U(t, r)=R(t, r)-P_{1}(r)-P_{2}(r) t, \quad V(t, r)=S(t, r)-P_{1}(r)+P_{2}(r) t .
$$


Thus by (3.6) and (3.7) we get the homogeneous initial condition

$$
U(0, r)=V(0, r)=U_{t}(0, r)=V_{t}(0, r)=0, \quad \forall r \in\left[r_{a}, r_{b}\right] .
$$

Moreover, system (3.3) is transformed into

$$
\left\{\begin{array}{l}
U_{t}+\frac{2 r t^{2}}{a^{\prime} \sqrt{a} V+f} U_{r}=\frac{U-V}{2 t}+b_{1}(U, V, t, r), \\
V_{t}-\frac{2 r t^{2}}{a^{\prime} \sqrt{a} U+g} V_{r}=\frac{V-U}{2 t}+b_{2}(U, V, t, r),
\end{array}\right.
$$

where $f=f(t, r)=a^{\prime} \sqrt{a}\left(P_{1}(r)-P_{2}(r) t\right)+2 r^{2} t, g=g(t, r)=a^{\prime} \sqrt{a}\left(P_{1}(r)+P_{2}(r) t\right)-2 r^{2} t$, and

$$
\begin{aligned}
b_{1}(U, V, t, r)= & \left(\frac{\left.a^{\prime} r^{2}\left[U+P_{1}(r)+P_{2}(r) t\right)\right]}{2 \sqrt{a}\left[a^{\prime} \sqrt{a} V+f\right]}-\frac{1}{2}\right)\left(\frac{U-V}{t}+2 P_{2}(r)\right) \\
& -\frac{2 t^{2} r}{a^{\prime} \sqrt{a} V+f}\left(P_{1}^{\prime}(r)+P_{2}^{\prime}(r) t\right), \\
b_{2}(U, V, t, r)= & \left(\frac{\left.a^{\prime} r^{2}\left[V-P_{1}(r)+P_{2}(r) t\right)\right]}{2 \sqrt{a}\left[a^{\prime} \sqrt{a} U+g\right]}-\frac{1}{2}\right)\left(\frac{V-U}{t}-2 P_{2}(r)\right) \\
& -\frac{2 t^{2} r}{a^{\prime} \sqrt{a} U+g}\left(P_{1}^{\prime}(r)-P_{2}^{\prime}(r) t\right) .
\end{aligned}
$$

Then the previous problem is reformulated as follows.

Problem 3.1 Under the assumptions in Theorem 1, we seek a classical solution to initial data problem (3.9) (3.10) in the region $t>0$.

To solve Problem (3.1), we first define a suitable function space. Let $\delta$ be a small positive constant. Set

$$
D(\delta):=\left\{(t, r) \mid 0 \leq t \leq \delta, r_{1}(t) \leq r \leq r_{2}(t)\right\}
$$

where $r_{1}(t), r_{2}(t)$ are continuously differentiable on $0 \leq t \leq \delta, r_{1}(0)=r_{a}, r_{2}(0)=r_{b}$ and $r_{1}(t)<r_{2}(t)$ for $0 \leq t \leq \delta$.

Definition 3.1 The domain $D(\delta)$ is called a strong domain of determinacy to system (3.10) if for any $(\xi, \eta) \in D(\delta)$ and any smooth functions $(U, V)$ satisfying the homogeneous initial condition (3.9), the curves $r_{ \pm}(t ; \xi, \eta)$ defined by

$$
\left\{\begin{array} { l } 
{ \frac { \mathrm { d } } { \mathrm { d } t } r _ { + } = \Lambda _ { + } ( V ( t , r _ { + } ) ) , } \\
{ r _ { + } | _ { t = \xi } = \eta , }
\end{array} \quad \left\{\begin{array}{l}
\frac{\mathrm{d}}{\mathrm{d} t} r_{-}=\Lambda_{-}\left(U\left(t, r_{-}\right)\right), \\
\left.r_{-}\right|_{t=\xi}=\eta,
\end{array}\right.\right.
$$

are also inside $D(\delta)$ for $0<t \leq \xi$. Here

$$
\Lambda_{+}(V)=\frac{2 r t^{2}}{a^{\prime} \sqrt{a} V+f}, \quad \Lambda_{-}(U)=-\frac{2 r t^{2}}{a^{\prime} \sqrt{a} U+g} .
$$

Next we define a suitable class of functions. Let $S=S(M, \delta)$ be a function class consisting of all continuously differentiable functions $F=\left(f_{1}, f_{2}\right)^{T}: D(\delta) \rightarrow \mathbb{R}^{2}$ satisfying the following properties 
$\left(S_{1}\right) f_{1}(0, r)=f_{2}(0, r)=\partial_{t} f_{1}(0, r)=\partial_{t} f_{2}(0, r)=0$,

$\left(S_{2}\right)\|\| \frac{f_{1}}{t^{2}}\left\|_{L^{\infty}}+\right\| \frac{f_{2}}{t^{2}} \|_{L^{\infty}} \leq M$,

$\left(S_{3}\right)\left\|\frac{\partial_{r} f_{1}}{t^{2}}\right\|_{L^{\infty}}+\left\|\frac{\partial_{r} f_{2}}{t^{2}}\right\|_{L^{\infty}} \leq M$,

$\left(S_{4}\right) \quad \partial_{r} F$ is Lipschitz continuous with respect to $r$ with $\left\|\frac{\partial_{r r}^{2} f_{1}}{t^{2}}\right\|_{L^{\infty}}+\left\|\frac{\partial_{r r}^{2} f_{2}}{t^{2}}\right\|_{L^{\infty}} \leq M$,

where $\delta$ and $M$ are two positive constants. Denote by $\mathcal{W}$ the function class containing only continuous functions on $D(\delta)$ satisfying $\left(S_{1}\right)$ and $\left(S_{2}\right)$. It is easy to see that $\mathcal{S}$ and $\mathcal{W}$ are subsets of $C^{0}\left(D(\delta) ; \mathbb{R}^{2}\right)$. Moreover, we define a weighted metric on $\mathcal{S}$ and $\mathcal{W}$ as follows:

$$
d(\mathbf{F}, \mathbf{G}):=\left\|\frac{f_{1}-g_{1}}{t^{2}}\right\|_{L^{\infty}}+\left\|\frac{f_{2}-g_{2}}{t^{2}}\right\|_{L^{\infty}} .
$$

It is not difficult to check that $(\mathcal{W}, d)$ is a completed metric space, while $(\mathcal{S}, d)$ is not a closed subset in $(\mathcal{W}, d)$.

Theorem 1 follows directly from the following theorem.

Theorem 3 Suppose that the conditions listed in Theorem 1 hold and that $D\left(\delta_{0}\right)$ is a strong domain of determinacy to the system (3.10) for some positive constant $\delta_{0}$. Then there exit constants $\delta \in\left(0, \delta_{0}\right)$ and $M$ such that the degenerate hyperbolic problem (3.10) and (3.9) has a classical solution in the function class $\mathcal{S}(M, \delta)$.

\section{Proof of the main theorems}

In this section, we use the fixed point method to prove Theorem 3 and then complete the proof of Theorem 1. The proof is divided into five steps. In Step 1, we construct an integration iteration mapping in the function class $\mathcal{S}(M, \delta)$ by the differential system (3.10). In Step 2, we establish a series of a priori estimates for $b_{1}, b_{2}$ and $\Lambda_{ \pm}$. We show that the above iteration mapping is a contraction in Step 3. In Step 4, we show that this limit vector function also belongs to $\mathcal{S}(M, \delta)$. Finally, in Step 5 we return the solution to the original coordinates $(r, \theta)$.

Step 1. The iteration mapping. Denote

$$
\frac{d}{d_{+}(V)}:=\partial_{t}+\Lambda_{+}(V) \partial_{r}, \quad \frac{d}{d_{-}(U)}:=\partial_{t}+\Lambda_{-}(U) \partial_{r} .
$$

Then system (3.10) can be rewritten as

$$
\frac{d}{d_{+}(V)} U=\frac{U-V}{2 t}+b_{1}(U, V, r, t), \quad \frac{d}{d_{-}(U)} V=\frac{V-U}{2 t}+b_{2}(U, V, r, t) .
$$

Assume the vector functions $(u, v)^{T}(t, r)$ are in the set $\mathcal{S}$, we consider the linear system of (3.11)

$$
\frac{d}{d_{+}(v)} U=\frac{u-v}{2 t}+b_{1}(u, v, r, t), \quad \frac{d}{d_{-}(u)} V=\frac{v-u}{2 t}+b_{2}(u, v, r, t),
$$

which combined with the property $S_{1}$ gives

$$
U(\xi, \eta)=\int_{0}^{\xi}\left\{\frac{u-v}{2 t}+b_{1}\left(t, r_{+}(t ; \xi, \eta)\right)\right\} \mathrm{d} t,
$$




$$
V(\xi, \eta)=\int_{0}^{\xi}\left\{\frac{v-u}{2 t}+b_{2}\left(t, r_{-}(t ; \xi, \eta)\right)\right\} \mathrm{d} t,
$$

where $r_{ \pm}$are defined as in (3.11) and

$$
\begin{aligned}
& b_{1}\left(t, r_{+}(t ; \xi, \eta)\right)=b_{1}\left(u\left(t, r_{+}(t ; \xi, \eta)\right), v\left(t, r_{+}(t ; \xi, \eta)\right), t, r_{+}(t ; \xi, \eta)\right) \\
& b_{2}\left(t, r_{-}(t ; \xi, \eta)\right)=b_{2}\left(u\left(t, r_{-}(t ; \xi, \eta)\right), v\left(t, r_{-}(t ; \xi, \eta)\right), t, r_{-}(t ; \xi, \eta)\right) .
\end{aligned}
$$

It is clear that Eqs. (4.4) and (4.5) define a mapping

$$
\mathcal{T}\left(\left(\begin{array}{l}
u \\
v
\end{array}\right)\right)=\left(\begin{array}{l}
U \\
V
\end{array}\right)
$$

Therefore, our problem is changed to find a fixed point of the mapping $\mathcal{T}$ in the set $\mathcal{S}(M, \delta)$ for some suitable constants $M$ and $\delta$.

Step 2. A priori estimates. We derive a series of estimates about $b_{1}, b_{2}$ and $\Lambda_{ \pm}$for later use. We will use $K>1$ to denote a constant depending only on $C^{3}$ norms of $a, P_{1}, P_{2}, \varphi^{\prime}$ and $k_{0}, k_{1}, k_{2}, r_{a}, r_{b}$. Since $(u, v)^{T} \in \mathcal{S}$, we see by (2.11) and (3.5) that there exists a small constant $\delta_{0}$ such that for $t \leq \delta_{0}$

$$
\left|a^{\prime} \sqrt{a} v+f\right| \geq \frac{k_{0} k_{1} k_{2}}{2} \geq \frac{1}{K}, \quad\left|a^{\prime} \sqrt{a} u+g\right| \geq \frac{k_{0} k_{1} k_{2}}{2} \geq \frac{1}{K} .
$$

Moreover, we have

$$
|u-v| \leq M t^{2}, \quad\left|u_{r}-v_{r}\right| \leq M t^{2}, \quad\left|u_{r r}-v_{r r}\right| \leq M t^{2} .
$$

To estimate $b_{1}$, we first note that

$$
\begin{aligned}
& \frac{a^{\prime} r^{2}\left[u+P_{1}(r)+P_{2}(r) t\right]}{2 \sqrt{a}\left(a^{\prime} \sqrt{a} v+f\right)}-\frac{1}{2} \\
& =\frac{\sqrt{a} r^{2}\left(a^{\prime} \sqrt{a} u+g+2 r^{2} t\right)-\sqrt{a}(a \sqrt{a} v+f)}{2 \sqrt{a}\left(a^{\prime} \sqrt{a} v+f\right)} \\
& =t \cdot \frac{t \cdot \frac{a a^{\prime}(u-v)}{t^{2}}+2 a a^{\prime} P_{2}(r)+a^{\prime} t\left[u+P_{1}(r)+P_{2}(r) t\right]-2 \sqrt{a} r^{2} t}{2 \sqrt{a}\left(a^{\prime} \sqrt{a} v+f\right)},
\end{aligned}
$$

from which one has

$$
\left|\frac{a^{\prime} r^{2}\left[u+P_{1}(r)+P_{2}(r) t\right]}{2 \sqrt{a}\left(a^{\prime} \sqrt{a} v+f\right)}-\frac{1}{2}\right| \leq t K \cdot(1+M t) .
$$

In addition, differentiating (4.8) with respect to $r$ obtains

$$
\begin{aligned}
& \left|\partial_{r}\left(\frac{a^{\prime} r^{2}\left[u+P_{1}(r)+P_{2}(r) t\right]}{2 \sqrt{a}\left(a^{\prime} \sqrt{a} v+f\right)}-\frac{1}{2}\right)\right| \leq t K \cdot(1+M t), \\
& \left|\partial_{r r}\left(\frac{a^{\prime} r^{2}\left[u+P_{1}(r)+P_{2}(r) t\right]}{2 \sqrt{a}\left(a^{\prime} \sqrt{a} v+f\right)}-\frac{1}{2}\right)\right| \leq t K \cdot(1+M t) .
\end{aligned}
$$


Furthermore, we denote the last term in $b_{1}$ by $\Phi=\frac{2 r t^{2}}{a^{\prime} \sqrt{a} v+f}\left(P_{1}^{\prime}(r)+P_{2}^{\prime}(r) t\right)$ and then obtain

$$
|\Phi|+\left|\Phi_{r}\right|+\left|\Phi_{r r}\right| \leq K t^{2}(1+M t)^{2}
$$

Combining (4.9)-(4.12) and using the expression of $b_{1}$ yield

$$
\left|b_{1}\right|+\left|b_{1 r}\right|+\left|b_{1 r r}\right| \leq K t(1+M t)^{2}
$$

By similar arguments for $b_{2}$ get

$$
\left|b_{2}\right|+\left|b_{2 r}\right|+\left|b_{2 r r}\right| \leq K t(1+M t)^{2}
$$

For $\Lambda_{+}$, we use the fact

$$
\left|a^{\prime} \sqrt{a} v+f\right|+\left|\partial_{r}\left(a^{\prime} \sqrt{a} v+f\right)\right|+\left|\partial_{r r}\left(a^{\prime} \sqrt{a} v+f\right)\right| \leq K(1+M t),
$$

to obtain

$$
\left|\Lambda_{+}\right|+\left|\partial_{r} \Lambda_{+}\right|+\left|\partial_{r r} \Lambda_{+}\right| \leq K t^{2}(1+M t)^{2} .
$$

Similarly, one has

$$
\left|\Lambda_{-}\right|+\left|\partial_{r} \Lambda_{-}\right|+\left|\partial_{r r} \Lambda_{-}\right| \leq K t^{2}(1+M t)^{2}
$$

Step 3. Contraction of the mapping. We have the following lemma.

Lemma 4.1 Under the assumptions of Theorem 3, there exist positive constants $\delta, M$ and $0<\beta<1$ such that

(1) $\mathcal{T}$ maps $\mathcal{S}$ into $\mathcal{S}$;

(2) for any pair $\mathbf{F}, \hat{\mathbf{F}}$ in $\mathcal{S}$,

$$
d(\mathcal{T}(\mathbf{F}), \mathcal{T}(\hat{\mathbf{F}})) \leq \beta d(\mathbf{F}, \hat{\mathbf{F}})
$$

Here the constants $M, \delta, \beta$ depend only on the $C^{3}$ norms of $a, P_{1}(r), P_{2}(r), \varphi^{\prime}$ and $k_{0}, k_{1}, k_{2}$, $r_{a}, r_{b}$.

Proof Let $\mathbf{F}=(u, v), \hat{\mathbf{F}}=(\hat{u}, \hat{v})$ be in set $\mathcal{S}$ and $\mathbf{G}=\mathcal{T}(\mathbf{F})=(U, V)$ and $\hat{G}=\mathcal{T}(\hat{\mathbf{F}})=(\hat{U}, \hat{V})$. It is obvious that $U(0, \eta)=V(0, \eta)=0$.

Moreover, we use (4.7) and (4.13)-(4.14) to obtain

$$
\begin{aligned}
& |U| \leq \int_{0}^{\xi}\left\{\left|\frac{u-v}{2 t}\right|+\left|b_{1}\right|\right\} \mathrm{d} t \leq \int_{0}^{\xi} \frac{M t}{2}+K t(1+M \delta)^{2} \mathrm{~d} t \leq \frac{M}{4} \xi^{2}+K \xi^{2}(1+M \delta)^{2}, \\
& |V| \leq \int_{0}^{\xi}\left\{\left|\frac{v-u}{2 t}\right|+\left|b_{2}\right|\right\} \mathrm{d} t \leq \int_{0}^{\xi} \frac{M t}{2}+K t(1+M \delta)^{2} \mathrm{~d} t \leq \frac{M}{4} \xi^{2}+K \xi^{2}(1+M \delta)^{2},
\end{aligned}
$$

from which we have

$$
\left|\frac{U(\xi, \eta)}{\xi^{2}}\right|+\left|\frac{V(\xi, \eta)}{\xi^{2}}\right| \leq \frac{M}{2}+K(1+M \delta)^{2} .
$$


In order to establish the bound of $U_{r} / t^{2}$, we differentiate (4.4) with respect to $\eta$ to find that

$$
\frac{\partial U}{\partial \eta}(\xi, \eta)=\int_{0}^{\xi}\left(\frac{u_{r}-v_{r}}{2 t}+\frac{\partial b_{1}}{\partial r}\right) \cdot \frac{\partial r_{+}}{\partial \eta} \mathrm{d} t
$$

where

$$
\frac{\partial r_{+}}{\partial \eta}(t ; \xi, \eta)=\exp \left\{\int_{\xi}^{t} \frac{\partial \Lambda_{+}(v)}{\partial r}\left(\tau, r_{+}(\tau ; \xi, \eta)\right) \mathrm{d} \tau\right\}
$$

Applying (4.7), (4.13) and (4.15), we derive

$$
\begin{aligned}
\left|\frac{\partial U}{\partial \eta}\right| & \leq \int_{0}^{\xi}\left(\left|\frac{u_{r}-v_{r}}{2 t}\right|+\left|\frac{\partial b_{1}}{\partial r}\right|\right) \cdot\left|\frac{\partial r_{+}}{\partial \eta}\right| \mathrm{d} t \\
& \leq \int_{0}^{\xi}\left(\frac{M}{2} t+K t(1+M \delta)^{2}\right) \exp \left\{K t^{3}(1+M \delta)^{2}\right\} \mathrm{d} t \\
& \leq \xi^{2}\left(\frac{M}{4}+K(1+M \delta)^{2}\right) \exp \left\{K \delta^{3}(1+M \delta)^{2}\right\} .
\end{aligned}
$$

A similar estimate holds for $V$. Hence we arrive at

$$
\left|\frac{U_{\eta}}{\xi^{2}}\right|+\left|\frac{V_{\eta}}{\xi^{2}}\right| \leq\left(\frac{M}{2}+K(1+M \delta)^{2}\right) \exp \left\{K \delta^{3}(1+M \delta)^{2}\right\} .
$$

To estimate $U_{r} / t^{2}$ and $V_{r} / t^{2}$, we differentiate (4.19) with respect to $\eta$ to obtain

$$
\frac{\partial^{2} U}{\partial \eta^{2}}(\xi, \eta)=\int_{0}^{\xi}\left\{\left(\frac{u_{r r}-v_{r r}}{2 t}+\frac{\partial^{2} b_{1}}{\partial r^{2}}\right)\left(\frac{\partial r_{+}}{\partial \eta}\right)^{2}+\left(\frac{u_{r}-v_{r}}{2 t}+\frac{\partial b_{1}}{\partial r}\right) \frac{\partial^{2} r_{+}}{\partial \eta^{2}}\right\} \mathrm{d} t,
$$

where

$$
\frac{\partial^{2} r_{+}}{\partial \eta^{2}}=\frac{\partial r_{+}}{\partial \eta} \cdot \int_{\xi}^{t} \frac{\partial^{2} \Lambda_{+}}{\partial r^{2}} \cdot \frac{\partial r_{+}}{\partial \eta} \mathrm{d} \tau
$$

It follows by (4.15) and (4.20) that

$$
\left|\frac{\partial^{2} r_{+}}{\partial \eta^{2}}\right| \leq K \delta^{3}(1+M \delta)^{2} \exp \left\{K \delta^{3}(1+M \delta)^{2}\right\} .
$$

Therefore, we have

$$
\begin{aligned}
\left|\frac{\partial^{2} U}{\partial \eta^{2}}\right| & \leq \int_{0}^{\xi}\left\{\left(\left|\frac{u_{r r}-v_{r r}}{2 t}\right|+\left|\frac{\partial^{2} b_{1}}{\partial r^{2}}\right|\right)\left|\frac{\partial r_{+}}{\partial \eta}\right|^{2}+\left(\left|\frac{u_{r}-v_{r}}{2 t}\right|+\left|\frac{\partial b_{1}}{\partial r}\right|\right)\left|\frac{\partial^{2} r_{+}}{\partial \eta^{2}}\right|\right\} \mathrm{d} t \\
& \leq \int_{0}^{\xi}\left\{\left(\frac{M}{2} t+K t(1+M \delta)^{2}\right)\left[1+K \delta^{3}(1+M \delta)^{2}\right] \exp \left\{K \delta^{3}(1+M \delta)^{2}\right\} \mathrm{d} t\right. \\
& \leq \xi^{2}\left(\frac{M}{4}+K(1+M \delta)^{2}\right)\left[1+K \delta^{3}(1+M \delta)^{2}\right] \exp \left\{K \delta^{3}(1+M \delta)^{2}\right\} .
\end{aligned}
$$


In the same way we have the bound for $V_{\eta \eta}$

$$
\left|\frac{\partial^{2} V}{\partial \eta^{2}}\right| \leq \xi^{2}\left(\frac{M}{4}+K(1+M \delta)^{2}\right)\left[1+K \delta^{3}(1+M \delta)^{2}\right] \exp \left\{K \delta^{3}(1+M \delta)^{2}\right\}
$$

which together with (4.23) yields

$$
\left|\frac{U_{\eta \eta}}{\xi^{2}}\right|+\left|\frac{V_{\eta \eta}}{\xi^{2}}\right| \leq\left(\frac{M}{2}+K(1+M \delta)^{2}\right)\left[1+K \delta^{3}(1+M \delta)^{2}\right] \exp \left\{K \delta^{3}(1+M \delta)^{2}\right\}
$$

We choose $M \geq 16 K>16$ and then set $\delta \leq \min \left\{\delta_{0}, 1 / M\right\}$ to see that

$$
\begin{aligned}
& \left(\frac{M}{2}+K(1+M \delta)^{2}\right)\left[1+K \delta^{3}(1+M \delta)^{2}\right] \exp \left\{K \delta^{3}(1+M \delta)^{2}\right\} \\
& \leq\left(\frac{M}{2}+\frac{M}{4}\right)\left(1+\frac{1}{64}\right) \exp \left(\frac{1}{64}\right)<\frac{5}{6} M<M .
\end{aligned}
$$

Therefore, we combine (4.18), (4.21) and (4.24) to conclude that $\left(S_{2}\right)-\left(S_{4}\right)$ are preserved by the mapping $\mathcal{T}$.

To prove $\mathcal{T}(\mathbf{F}) \in \mathcal{S}$, it is enough to show that $U_{\xi}(0, \eta)=V_{\xi}(0, \eta)=0$. We differentiate (4.4) with respect to $\xi$ to arrive at

$$
\frac{\partial U}{\partial \xi}(\xi, \eta)=\frac{u-v}{2 \xi}+b_{1}+\int_{0}^{\xi}\left(\frac{u_{r}-v_{r}}{2 t}+\frac{\partial b_{1}}{\partial r}\right) \frac{\partial r_{+}}{\partial \xi} \mathrm{d} t
$$

where

$$
\frac{\partial r_{+}}{\partial \xi}(t ; \xi, \eta)=-\Lambda_{+}(\xi, \eta, V(\xi, \eta)) \cdot \frac{\partial r_{+}}{\partial \eta}(t ; \xi, \eta)
$$

It is easily seen by (4.7), (4.13), (4.15) and (4.20) that $U_{\xi}(0, \eta)=0$. Similarly, we have $V_{\xi}(0, \eta)=0$, which means that $\mathcal{T}$ maps $\mathcal{S}$ into itself.

Next we we show that the inequality (4.17) holds for some positive constant $\beta<1$. According to the definition of the mapping $\mathcal{T}$, we have

$$
\frac{d}{d_{+}(v)} U=\frac{u-v}{2 t}+b_{1}(u, v, t, r), \quad \frac{d}{d_{+}(\hat{v})} \hat{U}=\frac{\hat{u}-\hat{v}}{2 t}+b_{1}(\hat{u}, \hat{v}, t, r),
$$

and from this and (4.1) one gets

$$
\begin{aligned}
& \frac{d}{d_{+}(v)}(U-\hat{U}) \\
& \quad=\frac{(u-\hat{u})-(v-\hat{v})}{2 t}+\left[b_{1}(u, v, t, r)-b_{1}(\hat{u}, \hat{v}, t, r)\right]+\left(\Lambda_{+}(v)-\Lambda_{+}(\hat{v})\right) \partial_{r} \hat{U} .
\end{aligned}
$$

Recalling the expression of $b_{1}$ suggests

$$
\begin{aligned}
& b_{1}(u, v, t, r)-b_{1}(\hat{u}, \hat{v}, t, r) \\
& \quad=\left(\frac{a^{\prime} r^{2}\left[u+P_{1}(r)+P_{2}(r) t\right]}{2 \sqrt{a}\left(a^{\prime} \sqrt{a} v+f\right)}-\frac{1}{2}\right)\left(\frac{u-\hat{u}}{t}-\frac{v-\hat{v}}{t}\right)
\end{aligned}
$$




$$
\begin{aligned}
& +\left(\frac{\hat{u}-\hat{v}}{t}+2 P_{2}(r)\right)\left\{\frac{a^{\prime} r^{2}\left[u+P_{1}(r)+P_{2}(r) t\right]}{2 \sqrt{a}\left(a^{\prime} \sqrt{a} v+f\right)}-\frac{a^{\prime} r^{2}\left[\hat{u}+P_{1}(r)+P_{2}(r) t\right]}{2 \sqrt{a}\left(a^{\prime} \sqrt{a} \hat{v}+f\right)}\right\} \\
& -(\Phi(v)-\Phi(\hat{v})):=I+I I+I I I .
\end{aligned}
$$

For $I$, we find by (4.9) that

$$
|I| \leq K t(1+M \delta)\left(\left|\frac{u-\hat{u}}{t}\right|-\left|\frac{v-\hat{v}}{t}\right|\right) \leq K t^{2}(1+M \delta) d(\mathbf{F}, \hat{\mathbf{F}}) .
$$

For $I I$, one has

$$
\begin{aligned}
|I I| & \leq K(1+M \delta)\left|\frac{u+P_{1}(r)+P_{2}(r) t}{a^{\prime} \sqrt{a} v+f}-\frac{\hat{u}+P_{1}(r)+P_{2}(r) t}{a^{\prime} \sqrt{a} \hat{v}+f}\right| \\
& =K(1+M \delta)\left|\frac{\left(a^{\prime} \sqrt{a} \hat{v}+f\right)(u-\hat{u})-\left[a^{\prime} \sqrt{a}\left(P_{1}+P_{2} t\right)+\hat{u}\right](v-\hat{v})}{\left(a^{\prime} \sqrt{a} v+f\right)\left(a^{\prime} \sqrt{a} \hat{v}+f\right)}\right| \\
& \leq K t^{2}(1+M \delta) d(\mathbf{F}, \hat{\mathbf{F}}) .
\end{aligned}
$$

By the definition of $\Phi$ in (4.12), it is easy to obtain

$$
|I I I|=|\Phi(v)-\Phi(\hat{v})| \leq K t^{4} d(\mathbf{F}, \hat{\mathbf{F}}) .
$$

Putting (4.30)-(4.32) into (4.29) yields

$$
\left|b_{1}(u, v, t, r)-b_{1}(\hat{u}, \hat{v}, t, r)\right| \leq K t^{2}(1+M \delta) d(\mathbf{F}, \hat{\mathbf{F}}) .
$$

In addition, we use the definition of $\Lambda_{+}$to obtain

$$
\left|\Lambda_{+}(v)-\Lambda_{+}(\hat{v})\right|=\left|\frac{2 r t^{2}}{a^{\prime} \sqrt{a} v+f}-\frac{2 r t^{2}}{a^{\prime} \sqrt{a} \hat{v}+f}\right| \leq K t^{4} d(\mathbf{F}, \hat{\mathbf{F}}) .
$$

Combining (4.28) and (4.33)-(4.34), we have

$$
\begin{aligned}
|U-\hat{U}| & \leq \int_{0}^{t}\left(\frac{t}{2}+K t^{2}(1+M \delta)+K M t^{6}\right) d(\mathbf{F}, \hat{\mathbf{F}}) \mathrm{d} t \\
& \leq t^{2}\left\{\frac{1}{4}+K \delta(1+M \delta)\right\} d(\mathbf{F}, \hat{\mathbf{F}}),
\end{aligned}
$$

from which one gets

$$
\left|\frac{U-\hat{U}}{t^{2}}\right| \leq\left\{\frac{1}{4}+K \delta(1+M \delta)\right\} d(\mathbf{F}, \hat{\mathbf{F}})
$$

Following the same argument as above one obtains for the estimate $|V-\hat{V}| / t^{2}$,

$$
\left|\frac{U-\hat{U}}{t^{2}}\right|+\left|\frac{V-\hat{V}}{t^{2}}\right| \leq\left\{\frac{1}{2}+2 K \delta(1+M \delta)\right\} d(\mathbf{F}, \hat{\mathbf{F}})=: \beta d(\mathbf{F}, \hat{\mathbf{F}})
$$

For choosing $\delta$ as before, we see that $\beta<1$, which concludes the proof of (4.17). The proof of Lemma 4.1 is complete. 
Step 4. Properties of the limit function. We claim that the limit of the iteration sequence $\mathbf{F}^{(n)}=\mathcal{T} \mathbf{F}^{(n-1)}$ is in the space $\mathcal{S}$. The claim follows directly from the lemma.

Lemma 4.2 Under the assumptions of Theorem 1, for the iteration sequence $\mathbf{F}^{(\mathbf{n})}, \partial_{t} \mathbf{F}^{(\mathbf{n})}(t, r)$ and $\partial_{r} \mathbf{F}^{(\mathbf{n})}(t, r)$ are uniformly Lipschitz continuous on $D(\delta)$.

Proof Assume $(u, v)^{T} \in \mathcal{S}$, we know by Lemma 4.1 that $(U, V)^{T}=\mathcal{T}(u, v)^{T}$ is also in $\mathcal{S}$. The proof is divided into three steps.

Firstly, we prove that $\left|U_{t}\right|+\left|V_{t}\right| \leq 2 M t$. This follows directly from (4.4) and (4.5). In fact, we recall the expression of $U_{\xi}$ given in (4.26) and use (4.7), (4.13) and (4.15) to obtain

$$
\begin{aligned}
\left|\frac{\partial U}{\partial \xi}\right| \leq & \left|\frac{u-v}{2 \xi}\right|+\left|b_{1}\right|+\int_{0}^{\xi}\left(\left|\frac{u_{r}-v_{r}}{2 t}\right|+\left|\frac{\partial b_{1}}{\partial r}\right|\right)\left|\frac{\partial r_{+}}{\partial \xi}\right| \mathrm{d} t \\
\leq & \frac{M \xi}{2}+K \xi(1+M \delta)^{2} \\
& +\int_{0}^{\xi}\left(\frac{M t}{2}+K t(1+M \delta)^{2}\right) \cdot K \delta^{2}(1+M \delta)^{2} \exp \left\{K \delta^{3}(1+M \delta)^{2}\right\} \mathrm{d} t \leq M
\end{aligned}
$$

for choosing $M$ and $\delta$ as in (4.25).

Secondly, we show that $\left|U_{t r}\right|+\left|V_{t r}\right| \leq 2 M t$. To prove it, we differentiate (4.19) with respect to $\xi$ to obtain

$$
\begin{aligned}
\frac{\partial^{2} U}{\partial \xi \partial \eta}= & \left(\frac{u_{r}-v_{r}}{2 \xi}+\frac{\partial b_{1}}{\partial r}\right) \frac{\partial r_{+}}{\partial \eta} \\
& +\int_{0}^{\xi}\left\{\left(\frac{u_{r r}-v_{r r}}{2 t}+\frac{\partial^{2} b_{1}}{\partial r^{2}}\right) \frac{\partial r_{+}}{\partial \eta} \frac{\partial r_{+}}{\partial \xi}+\left(\frac{u_{r}-v_{r}}{2 t}+\frac{\partial b_{1}}{\partial r}\right) \frac{\partial^{2} r_{+}}{\partial \xi \partial \eta}\right\} \mathrm{d} t,
\end{aligned}
$$

where

$$
\frac{\partial^{2} r_{+}}{\partial \xi \partial \eta}=\frac{\partial r_{+}}{\partial \eta}\left\{\int_{\xi}^{t} \frac{\partial^{2} \Lambda_{+}(v)}{\partial r^{2}} \cdot \frac{\partial r_{+}}{\partial \xi} \mathrm{d} \tau-\frac{\partial \Lambda_{+}(v)}{\partial r}\right\} .
$$

By employing (4.7), (4.13), (4.15), (4.20) and (4.27), we find that

$$
\begin{aligned}
\left|\frac{\partial^{2} U}{\partial \xi \partial \eta}\right| \leq & \left(\frac{M \xi}{2}+K \xi(1+M \delta)^{2}\right) \exp \left\{K \delta^{3}(1+M \delta)^{2}\right\} \\
& +\int_{0}^{\xi}\left(\frac{M t}{2}+K t(1+M \delta)^{2}\right) \exp \left\{2 K \delta^{3}(1+M \delta)^{2}\right\}\left(K t^{2}+K t^{2}(1+M \delta)^{2}\right) \mathrm{d} t \\
\leq & M \xi
\end{aligned}
$$

if $M$ and $\delta$ are chosen as in (4.25), from which and the corresponding estimate for $V_{\xi \eta}$ we have $\left|U_{\xi \eta}\right|+\left|V_{\xi \eta}\right| \leq 2 M \xi$.

Finally, we claim that $\left|U_{t t}\right|+\left|V_{t t}\right| \leq 10 M$. Differentiating (4.26) with respect to $\xi$ leads to

$$
\begin{aligned}
\frac{\partial^{2} U}{\partial \xi^{2}}= & \frac{u_{\xi}-v_{\xi}}{2 \xi}-\frac{u-v}{2 \xi^{2}}+\frac{\partial b_{1}}{\partial \xi}+\left(\frac{u_{r}-v_{r}}{2 \xi}+\frac{\partial b_{1}}{\partial r}\right) \frac{\partial r_{+}}{\partial \xi} \\
& +\int_{0}^{\xi}\left\{\left(\frac{u_{r r}-v_{r r}}{2 t}+\frac{\partial^{2} b_{1}}{\partial r^{2}}\right)\left(\frac{\partial r_{+}}{\partial \xi}\right)^{2}+\left(\frac{u_{r}-v_{r}}{2 t}+\frac{\partial b_{1}}{\partial r}\right) \frac{\partial^{2} r_{+}}{\partial \xi^{2}}\right\} \mathrm{d} t
\end{aligned}
$$


where

$$
\frac{\partial^{2} r_{+}}{\partial \xi^{2}}=-\frac{\partial \Lambda_{+}}{\partial \xi} \frac{\partial r_{+}}{\partial \eta}-\Lambda_{+} \frac{\partial^{2} r_{+}}{\partial \xi \partial \eta}
$$

By a direct calculation, one has

$$
\begin{aligned}
\left|\frac{\partial^{2} r_{+}}{\partial \xi^{2}}\right| & \leq K \xi(1+M \delta) \exp \left\{K \delta^{3}(1+M \delta)^{2}\right\}+K \xi^{4}(1+M \delta)^{2} \exp \left\{2 K \delta^{3}(1+M \delta)^{2}\right\} \\
& \leq 2 K \xi(1+M \delta) \exp \left\{K \delta^{3}(1+M \delta)^{2}\right\} .
\end{aligned}
$$

Furthermore, according the expression of $b_{1}$ arrives at

$$
\begin{aligned}
\frac{\partial b_{1}}{\partial t}= & \left(\frac{a^{\prime} r^{2}\left(u+P_{1}+P_{2} t\right)}{2 \sqrt{a}\left[a^{\prime} \sqrt{a} v+f\right]}-\frac{1}{2}\right)\left(\frac{u_{t}-v_{t}}{t}-\frac{u-v}{t^{2}}\right) \\
& +\frac{\partial}{\partial t}\left(\frac{a^{\prime} r^{2}\left(u+P_{1}+P_{2} t\right)}{2 \sqrt{a}\left[a^{\prime} \sqrt{a} v+f\right]}\right)\left(\frac{u-v}{t}+2 P_{2}\right) \\
& -\frac{2 t^{2} r P_{2}^{\prime}}{a^{\prime} \sqrt{a} v+f}-\frac{4 \operatorname{tr}\left(P_{1}^{\prime}+P_{2}^{\prime} t\right)}{a^{\prime} \sqrt{a} v+f}+\frac{2 t^{2} r\left(P_{1}^{\prime}+P_{2}^{\prime} t\right)}{\left(a^{\prime} \sqrt{a} v+f\right)^{2}} \partial_{t}\left(a^{\prime} \sqrt{a} v+f\right),
\end{aligned}
$$

from which and (4.7), (4.9) and the fact $\left|u_{t}\right|+\left|v_{t}\right| \leq 2 M t$ we obtain the estimate of $b_{1 t}$ by a straight forward calculation

$$
\left|\frac{\partial b_{1}}{\partial t}\right| \leq K(1+M \delta)^{3}
$$

Inserting the above and (4.37) into (4.36), we see that

$$
\begin{aligned}
\left|\frac{\partial^{2} U}{\partial \xi^{2}}\right| \leq & M+M+K(1+M \delta)^{3}+K \delta^{2}(M \delta+K \delta(1+M \delta)) \exp \left\{2 K \delta^{3}(1+M \delta)^{2}\right\} \\
& +K \delta^{2}(1+M \delta)^{2} \exp \left\{2 K \delta^{3}(1+M \delta)^{2}\right\} \leq 5 M
\end{aligned}
$$

for choosing $M$ and $\delta$ as in (4.25), i.e., $M \geq 16 K$ and $\delta \leq 1 / M$. Repetition of the same argument for $V$ obtains $\left|V_{\xi \xi}\right| \leq 5 M$, which together with (4.38) gets $\left|U_{\xi \xi}\right|+\left|V_{\xi \xi}\right| \leq 10 M$.

The proof of Lemma 4.2 is completed by Lemma 4.1 and the above estimates.

With the help of Lemma 4.1 and Lemma 4.2, we conclude Theorem 3.

Step 5. Convert solution back to original variables. We now return the solution in the coordinate plane $(t, \tilde{r})$ into the original coordinate plane $(r, \theta)$. By the definitions of $U$ and $V$ in (3.8), we first know the functions $R(t, \tilde{r})$ and $S(t, \tilde{r})$. In addition, we see that the coordinate transformation $(r, \theta) \mapsto(t, \tilde{r})$ is a one-to-one mapping. Indeed, the Jacobian is

$$
J=\frac{\partial(t, \tilde{r})}{\partial(r, \theta)}=\frac{a^{\prime}(P)\left[U+V+2 P_{1}(r)\right]}{4 t},
$$

which is strictly positive or strictly negative. Therefore, we can obtain $R$ and $S$ as functions of $r$ and $\theta$. We integrate the first equation of (2.9) to obtain the function $P(r, \theta)$. Thus the 
proof of Theorem 1 is complete. In order to conclude Theorem 2, it suffices to check that the relation $P_{r}=(R-S) /(2 \lambda)$ holds on $D(\delta)$. By a direct calculation, we find that

$$
\partial_{\theta}\left(R-S-2 \lambda P_{r}\right)=-\frac{a^{\prime}(P) r^{2}(R+S)}{4 a(P)\left[r^{2}-a(P)\right]}\left(R-S-2 \lambda P_{r}\right) .
$$

Since $P_{r}=P_{2}(r)$ is uniformly bounded and $R=S=P_{1}(r)$ on $\Gamma, G:=R-S-2 \lambda P_{r}=0$ on $\Gamma$. We note that, in terms of $(t, \tilde{r})$, Eq. (4.39) can be rewritten as

$$
\partial_{t} G=\frac{r^{2}}{a} \cdot \frac{G}{t}
$$

Moreover, we have

$$
\left.\frac{G}{t}\right|_{t=0}=\left.\frac{U-V+2 P_{2}(r) t-\frac{2 r t}{\sqrt{r^{2}-t^{2}}} P_{r}}{t}\right|_{t=0}=\left.\frac{U-V}{t}\right|_{t=0}=0,
$$

which together with (4.40) leads to $G \equiv 0$ on $D(\delta)$, which completes the proof of Theorem 2 .

\section{Acknowledgements}

The authors would like to thank the anonymous reviewers for their careful work and comments that helped to improve the clarity of the paper.

\section{Funding}

This work was supported by the NSF of Zhejiang Province of China (LY17A010019 and LY19A010012) and NSFC (11301128 and 11571088).

\section{Availability of data and materials}

Not applicable.

\section{Competing interests}

The authors declare that they have no competing interests.

\section{Authors' contributions}

All authors have contributed equally to the paper. All authors read and approved the final manuscript.

\section{Publisher's Note}

Springer Nature remains neutral with regard to jurisdictional claims in published maps and institutional affiliations.

Received: 11 October 2018 Accepted: 25 December 2018 Published online: 05 January 2019

\section{References}

1. Bers, L.: Mathematical Aspects of Subsonic and Transonic Gas Dynamics. Wiley, New York (1958)

2. Bouhmadi-Lopez, M., Frazao, P., Henriques, A.: Stochastic gravitational waves from a new type of modified Chaplygin gas. Phys. Rev. D 81, 063504 (2010)

3. Čanié, S., Keyfitz, B., Kim, E.: Mixed hyperbolic-elliptic systems in self-similar flows. Bol. Soc. Bras. Mat. 32, 1-23 (2002)

4. Čanié, S., Keyfitz, B., Kim, E.: Free boundary problems for nonlinear wave systems: Mach stems for interacting shocks. SIAM J. Math. Anal. 37, 1947-1977 (2006)

5. Chaplygin, S.: On gas jets. Sci. Mem. Moscow Univ. Math. Phys. 21, 1-121 (1904)

6. Chen, G., Deng, X., Xiang, W.: Shock diffraction by convex cornered wedges for the nonlinear wave system. Arch. Ration. Mech. Anal. 211, 61-112 (2014)

7. Glimm, G., Ji, X., Li, J., Li, X., Zhang, P., Zhang, T., Zheng, Y.: Transonic shock formation in a rarefaction Riemann problem for the 2-D compressible Euler equations. SIAM J. Appl. Math. 69, 720-742 (2008)

8. Gorini, V., Kamenshchik, A., Moschella, U., Pasquier, V.: The Chaplygin gas as a model for dark energy. arXiv:gr-qc/0403062

9. $\mathrm{Hu}, \mathrm{Y} ., \mathrm{Li}, \mathrm{J}$.: Sonic-supersonic solutions for the two-dimensional steady full Euler equations (2017, submitted)

10. Hu, Y., Wang, G.: Semi-hyperbolic patches of solutions to the two-dimensional nonlinear wave system for Chaplygin gases. J. Differ. Equ. 257, 1567-1590 (2014)

11. Hu, Y., Wang, G.: The interaction of rarefaction waves of a two-dimensional nonlinear wave system. Nonlinear Anal., Real World Appl. 22, 1-15 (2015) 
12. Jang, J., Kim, E.: Diffraction of a shock into an expansion wavefront for the transonic self-similar nonlinear wave system in two space dimensions. J. Differ. Equ. 260, 445-477 (2016)

13. Jegdic, K., Keyfitz, B., Čanié, S.: Transonic regular reflection for the nonlinear wave system. J. Hyperbolic Differ. Equ. 3 443-474 (2006)

14. Kim, E.: A global subsonic solution to an interacting transonic shock for the self-similar nonlinear wave equation. J. Differ. Equ. 248, 2906-2930 (2010)

15. Kim, E: An interaction of a rarefaction wave and a transonic shock for the self-similar two-dimensional nonlinear wave system. Commun. Partial Differ. Equ. 37, 610-646 (2012)

16. Kim, E., Lee, C.: Transonic shock reflection problems for the self-similar two-dimensional nonlinear wave system. Nonlinear Anal. TMA 79, 85-102 (2013)

17. Lai, G., Sheng, W:: Centered wave bubbles with sonic boundary of pseudosteady Guderley Mach reflection configurations in gas dynamics. J. Math. Pures Appl. 104, 179-206 (2015)

18. Li, J., Sheng, W., Zhang, T., Zheng, Y.: Two-dimensional Riemann problems: from scalar conservation laws to compressible Euler equations. Acta Math. Sci. Ser. B Engl. Ed. 29, 777-802 (2009)

19. Li, J., Zhang, T., Yang, S.: The Two-Dimensional Riemann Problem in Gas Dynamics. Longman, Harlow (1998)

20. Li, J., Zhang, T., Zheng, Y.: Simple waves and a characteristic decomposition of the two dimensional compressible Euler equations. Commun. Math. Phys. 267, 1-12 (2006)

21. Li, J., Zheng, Y.: Interaction of rarefaction waves of the two-dimensional self-similar Euler equations. Arch. Ration. Mech. Anal. 193, 623-657 (2009)

22. Li, J., Zheng, Y.: Interaction of four rarefaction waves in the bi-symmetric class of the two-dimensional Euler equations. Commun. Math. Phys. 296, 303-321 (2010)

23. Li, M., Zheng, Y.: Semi-hyperbolic patches of solutions of the two-dimensional Euler equations. Arch. Ration. Mech. Anal. 201, 1069-1096 (2011)

24. Li, T., Yu, W.: Boundary Value Problem for Quasilinear Hyperbolic Systems. Duke University (1985)

25. Sheng, W., You, S.: Interaction of a centered simple wave and a planar rarefaction wave of the two-dimensional Euler equations for pseudo-steady compressible flow. J. Math. Pures Appl. 114, 29-50 (2018)

26. Song, K., Wang, Q., Zheng, Y.: The regularity of semihyperbolic patches near sonic lines for the 2-D Euler system in gas dynamics. SIAM J. Math. Anal. 47, 2200-2219 (2015)

27. Song, K., Zheng, Y.: Semi-hyperbolic patches of solutions of the pressure gradient system. Discrete Contin. Dyn. Syst. 24, 1365-1380 (2009)

28. Tsien, H.: Two dimensional subsonic flow of compressible fluids. J. Aeronaut. Sci. 6, 399-407 (1939)

29. Von Karman, T.: Compressibility effects in aerodynamics. J. Aeronaut. Sci. 8, 337-365 (1941)

30. Wang, Q., Zheng, Y.: The regularity of semi-hyperbolic patches at sonic lines for the pressure gradient equation in gas dynamics. Indiana Univ. Math. J. 63, 385-402 (2014)

31. Wang, R., Wu, Z.: Existence and uniqueness of solutions for some mixed initial boundary value problems of quasilinear hyperbolic systems in two independent variables. Acta Sci. Natur. Jilin Univ. 2, 459-502 (1963) (in Chinese)

32. Zhang, T., Zheng, Y:: Conjecture on the structure of solution of the Riemann problem for two-dimensional gas dynamics systems. SIAM J. Math. Anal. 21, 593-630 (1990)

33. Zhang, T., Zheng, Y.: Sonic-supersonic solutions for the steady Euler equations. Indiana Univ. Math. J. 63, 1785-1817 (2014)

34. Zhang, T., Zheng, Y:: The structure of solutions near a sonic line in gas dynamics via the pressure gradient equation. J. Math. Anal. Appl. 443, 39-56 (2016)

35. Zhang, T., Zheng, Y.: Existence of classical sonic-supersonic solutions for the pseudo steady Euler equations. Sci. Sin., Math. 47, 1-18 (2017) (in Chinese)

36. Zheng, Y: Systems of Conservation Laws: Two-Dimensional Riemann Problems. Birkhäuser, Boston (2001)

\section{Submit your manuscript to a SpringerOpen ${ }^{\circ}$ journal and benefit from:}

- Convenient online submission

- Rigorous peer review

- Open access: articles freely available online

- High visibility within the field

- Retaining the copyright to your article

Submit your next manuscript at $\gg$ springeropen.com 